\author{
Professor Melike BÍLDİRİİ, PhD \\ E-mail: melikebildirici@gmail.com \\ Department of Economics \\ Yıldız Technical University \\ Professor Fazıl KAYIKÇI, PhD \\ E-mail: fkayikci@yildiz.edu.tr \\ Department of Economics \\ Yıldız Technical University
}

\title{
THE RELATION BETWEEN GROWTH, ENERGY IMPORTS, MILITARIZATION AND CURRENT ACCOUNT BALANCE IN INDIA, BRAZIL, TURKEY AND PAKISTAN
}

\begin{abstract}
This paper analyzes the relationship among economic growth, current account balance, energy imports and militarization for the selected countries by using Markov Switching-Bayesian Vector Auto Regressive approach from 1972 to 2017. India, Brazil, Turkey and Pakistan are selected since they have higher rate of import of weapons, current account deficits, and high rates of energy imports. The results showed that the relation between the selected variables changes in these countries with respect to their energy and weapon import levels.. Energy imports increase in effect of militarization races and economic growth. The current account of the selected countries are vulnerable to the negative energy shocks and militarization.
\end{abstract}

Keywords: Militarization, Current Account Balance, Energy Imports.

JEL Classification : C22, F32, F41, F43, Q43.

\section{Introduction}

Militarization races, energy consumption, economic growth and current account balance are the significant macroeconomic variables that have crucial impacts on each other's. Especially, imports of military equipment and energy have substantial impact on the current account balance. Military and energy imports by using scarce foreign exchange reserves decrease available resources for the imports of intermediate and investment goods and distort the link between savings and investments. Furthermore, macroeconomic conditions of the countries are adversely affected from these conditions. Deteriorating effects on external accounts arise because of coercing the nations to exhaust their reserves. Also, borrowing from abroad leads to the crowding out of the tradable sector. Because the prices of military equipment and energy shocks have a significant influence on net foreign asset positions, these countries can experience a slowdown in their economic growth.

Increase in imports of energy and military weapons can cause to long-run fluctuations in the current account balances of the countries. In this perspective, this

DOI: $10.24818 / 18423264 / 55.3 .21 .03$ 
paper aims to investigate the dynamic and causal relationship among militarization, current account balance, energy imports and economic growth in India, Brazil, Turkey and Pakistan by using Markov Switching Bayesian Vector Auto Regressive (MSBVAR) and Markov Switching Bayesian Granger Causality (MSBGC) methods. These countries were selected since they have high level of imports of military weapons and energy. These countries have higher rate of import of weapons, current account deficits, and high rates of energy imports. India, Pakistan, and Turkey's economic growth are closely linked with the terrorist attacks in these countries. Imports of weapons and energy increases in these countries as a result of terrorist attacks. In this condition, determining the direction of causality among militarization, current account balance, energy imports, and economic growth became more important to determine the appropriate defense and energy policies for these economies.

Especially, the trade balances these in oil-importing countries are very important to observe the economic shocks and the transmission instruments [1]. Geopolitical, and the other extreme events such as Arab Spring and ISID terrorism, oligopolistic structure in refinery and redistribution, structure of market, military events, rapid demand growth were among the primary reasons of oil price [2] that have significant power of wealth redistribution between energy importing and energy exporting countries [3]. In the effect of the presented problems above, business cycles have to be taken into account, otherwise the estimated parameters would be improper and misrepresentative. To overcome these problems, the sample is divided into subsamples by considering the structural breaks in the analyzed time period; the researcher must describe these endogenously by depending on the data and the date of these fluctuations. Markov Switching (MS) method provides the various improvement to solve various problems such as incorrect parameter estimation in many papers. [4] attracted the attentions with his pioneering paper analyzed the volatility of oil price by MS method. MSBVAR and MSBGC methods that was employed by this paper will provide the various improvements to solve the above mentioned problems. This paper can be accepted as harmonizer of the earlier empirical papers. However, it diverges from the current literature with simultaneous estimations of the relation among militarization, energy import, current account and economic growth which was analyzed by MSBVAR and MSBGC methods. Furthermore, there are some papers investigated the nexus among economic growth, current account balance, and energy consumption, and the relation among economic growth, military expenditure and current account balance, nevertheless, there is no the literature of energy economics simultaneously analyzing the relation among energy consumption, militarization, current account balance, and economic growth although this relationship is so critical.

After introduction, the second section of the study mentions about the literature. The third section discusses theoretically the link among economic growth, military expenditure, oil imports and current account balance. The fourth section presents the econometric theory while the fifth section comprises the empirical 
The Relation Between Growth, Energy Imports, Militarization and Current Account Balance in India, Brazil, Turkey and Pakistan

results. Finally, the last section includes conclusions and economic policy implications.

\section{Literature Review}

[1] accepted that net foreign asset of oil importing countries tends to decline if oil demand tend to increase, but this decline has not always statistically significant. Their results showed that exchange rates and petroleum prices induce to trade imbalance and it was found the evidence of uni-directional causal relation from oil prices to trade imbalance. [5] found that higher government expenditure played an important role in the United States (US) trade balance in the 1970s and 1980s.

According to Keynesian view, known as Military Keynesianism, the military expenditures can be used to regulate the business cycle. [6], [7]. These opinions gained the importance after defense expenditure by India for armament purposes during the 1930s and military expenses making by the US before World War II. After 2000, the military fiscal policy was evaluated with the heavy reliance on military expenditures by the US to influence its business cycle [7]. [8] presented the DSGE approach estimated with the Bayesian technique for US economy and the found a positive military spending shock increases the US per capita real GDP growth.

Some papers tested the relationship between energy consumption and current account balance. [9] tested the causal relation among electricity consumption, current account balance, and economic growth in the period of 19812013 for China, India, Singapore, South Korea, Turkey, and Taiwan. [10] analyzed the relation between crude oil trade and current account of a country for 91 countries during the 1984-2009 period and it was presented that oil exports are a significant reason of enlightening current account surpluses nonetheless that oil imports have not impact on current account deficits.

[11] and [12] showed that military expenditure accelerates the economic growth. [6] determined that, during the 2002 and 2003, military expenditures help to increase the economic growth. Moreover, 60 percent of the economic growth during this period was originated by military expenditures. Some of the other papers searched the nexus between economic growth and military expenditures. They determined the existence of positive or negative effects on each other's.

The trademill of destruction theory found that the energy usage is positively related with militarization scale [13], [14] showed the nexus between military spending, energy usage, and real GDP. [15] found that military sector causes environmental degradation. [16] tested nexus between economic growth, petroleum consumption and militarization in BRICTSM countries for the period 1987- 2013 by using ARDL and causality approach. She found the evidence of bidirectional causal link among the variables. 


\section{The Military Equipment Imports and Energy Imports in the Selected Countries}

Militarization race accelerated after World War II and especially 2000, and it continues to increase. The imports of military equipment and energy create a vast burden on the economy, because of using scarce foreign reserves, which cause to trade deficits. The financial burden of military equipment imports grow over time. Increasing militarization resulted with an increase in energy consumption. On the one side, while the wealth shifted from weapon importing countries to producer countries, the increased oil price also reallocate the wealth between oil importing and exporting countries, because the price elasticity of demand for gasoline and oil are inelastic [17]. In oil-importing countries, an exogenous rise in the price of imported energy creates negative effect on trade by leading to economic shock which has the effects on production decisions. The increasing oil prices decreases the economic growth, but there is some uncertainty about the size of this effect [18]. The economic growth of the selected countries is closely linked to the country's militarization and energy usage, and energy usage of the rest of the world. Since imported energy is an intermediate input in the internal production and a rise in energy prices causes direct increase in the input cost. On the other hand, import of weapons and energy leads to an overall negative trade balance. Moreover, defense sector use huge amounts of oil [13-16] in military institutions, in their activities and in their weapons, ships, planes, and tanks. For example, approximately $25 \%$ of jet fuel was used by military institutions over the world in 1980's; and nearly threequarters of the oil were used by the vehicles, sea vessels, aircraft, and other warfare machinery of the armed forces [19].

\section{Data Description and Econometric Methodology \\ 4.1. Data Description}

The data was employed from 1972 to 2017 for all countries. The energy imports (C), militarization (ML), current account balance (CA) and real per Capita GDP (Y) data were employed. All data were logged (ln) to minimize skewness. Energy imports (\% of total energy use) data was taken from IEA energy statistics. The military import data (ML) is measured in constant 2005\$ and obtained from SIPRI [20]. Real per Capita GDP (constant 2010\$) and Current account data (\% of GDP) were obtained from World Bank.

\subsection{Econometric Methodology}

\subsubsection{MS-Bayesian VAR Analysis}

[4], as an alternative to a stationary linear autoregressive model, proposed a simple nonlinear framework for economic time series with a permanent and cyclical component.

The MSI(.)-VAR(.) model is:

$$
y_{t}=\mu\left(s_{t}\right)+\sum_{k=1}^{q} A_{k}\left[\left(y_{t-k}-\mu\left(s_{t-k}\right)\right]+u_{t}\right. \text {, }
$$


The Relation Between Growth, Energy Imports, Militarization and Current Account Balance in India, Brazil, Turkey and Pakistan

$$
u_{t} / s_{t} \sim N I D\left(0, \sum\left(s_{t}\right)\right) . \text { And } \mu\left(s_{t}\right), A_{k}\left(s_{t}\right) \text { are parameter shift functions }
$$

that describe the dependence of the parameters $\mu(t), A_{k}$ on the realized regime $s_{t}$. The MSI(M)-VAR(p) is

$$
\begin{aligned}
& y_{t}=c\left(s_{t}\right)+\sum_{k=1}^{p} A_{k} y_{t-k}+u_{t} \\
& u_{t} / s_{t} \sim N I D\left(0, \sum\left(s_{t}\right)\right) . A_{i}(.) \text { represents the coefficients of the variables' }
\end{aligned}
$$

lagged values in different regimes and $\sum$ represents the variances of the residuals for each regime. $\mu\left(s_{t}\right)$ defines the dependence of the mean $\mu$ of the $\mathrm{K}$ dimensional time series vector on the regime variable $s_{t}$.

In an MS-VAR model, Markov chain governs $s_{t}$, and the probability that the state variable equals some particular value $\mathrm{j}$ depends on the most recent value $\mathrm{s}_{\mathrm{t}-1}$ :

$$
P\left\{s_{t}=j \mid s_{t-1}=i, s_{t-2}=k, \ldots \ldots \ldots . . .\right\}=P\left\{s_{t}=j \mid s_{t-1}=i\right\}=p_{i j}
$$

As such, a structure may prevail for a random period of time, and will be replaced by another structure when switching takes place. The transition probability $\mathrm{p}_{\mathrm{ij}}$ gives the probability that state $\mathrm{i}$ will be followed by state $\mathrm{j}$. Thus, the transition probabilities satisfy

$$
p_{i 1}+p_{i 2}+\ldots \ldots \ldots+p_{i N}=1
$$

It is assumed that $s$ follows an irreducible ergodic M state Markov process with the transition matrix defined as,

$$
\mathbf{P}=\left[\begin{array}{cccc}
p_{11} & p_{12} & \cdots & p_{1 M} \\
p_{21} & p_{22} & \cdots & p_{2 M} \\
\vdots & \vdots & \cdots & \vdots \\
p_{M 1} & p_{M M} & \cdots & p_{M M}
\end{array}\right]
$$

To estimate the MS models, there are different methods, such as the maximum likelihood estimate (MLE) and the expectation maximization (EM) suggested by Hamilton. The EM algorithm has been designed to estimate the parameters of a model where the observed time series depends on an unobserved or a hidden stochastic variable. To make inference, it was used the iterative method for $\mathrm{t}=1,2$, $\mathrm{T}$, while taking the previous value of this probability,

$$
\xi_{i t-1}=P_{r}\left[s_{t-1}=i \mid \Omega_{t-1} ; \alpha\right] \quad \text { as input }
$$

This inference can be demonstrated as

$$
\xi_{\text {it }}=P_{r}\left[S_{t}=i \mid \Omega_{t} ; \alpha\right]
$$


where $\mathrm{i}=1,2 ; \Omega_{t}$ stands for information set and $\theta$ is the vector of parameters to be estimated.

The iterative estimation technique can be used to make inference as

$$
\xi_{i t-1}=P_{r}\left[s_{t-1}=i \mid \Omega_{t-1} ; \alpha\right] \quad \text { for } t=1,2, \ldots, T
$$

The conditional log likelihood can be given as

$$
\log f\left(y_{1}, y_{2}, \ldots, y_{T} \mid y_{0} ; \alpha\right)=\sum \log f\left(y_{t} \mid \Omega_{t-1} ; \alpha\right)
$$

The MSBVAR Granger causality methodology can be given as follows.

$$
\left[\begin{array}{l}
d y_{t} \\
d x_{t}
\end{array}\right]=\left[\begin{array}{l}
n_{1, s t} \\
n_{2, s t}
\end{array}\right]+\sum_{k=1}^{q}\left[\begin{array}{ll}
\varphi_{11, s t}^{(k)} & \varphi_{12, s t}^{(k)} \\
\varphi_{21, s t}^{(k)} & \varphi_{22, s t}^{(k)}
\end{array}\right]\left[\begin{array}{l}
d y_{t-k} \\
d x_{t-k}
\end{array}\right]+\left[\begin{array}{c}
e_{t} \\
\varepsilon_{t}
\end{array}\right]
$$

When any of the coefficients of $d y_{t-1}, \ldots, \mathrm{dy}_{t-q}$ in regimes is different from 0 in the equation for $d x$, it is accepted that $d y$ is Granger cause of $d x$ in that regime. Granger causality can be identified by testing $H_{0}: \varphi_{12}{ }^{(k)}=0$ and $H_{0}: \varphi_{21}{ }^{(k)}=0$. The approach needs the estimation of either MSIAH(.)BVAR(.) or MSIA(.)BVAR(.) model.

The Bayesian inference that is dependent upon the posterior distribution of $\theta$ is employed (see [21, 22] for detailed information). The likelihood function is given as

$$
p(\alpha)=\prod_{i=1}^{N} p\left(\alpha_{i}\right) p(P)
$$

This state permits the possibility of prior knowledge of the state-specific parameters, $\alpha_{\mathrm{st}}$, individually for each regime.

The posterior distribution is demonstrated as

$$
p(\alpha \mid y, S) \infty \prod_{i=1}^{N} p\left(\alpha_{i} \mid y, S\right) p(P \mid y, S)
$$

And it can be decomposed into a posterior density of the transition probabilities matrix:

$$
p(P \mid S) \infty \prod_{i=1}^{N} p\left(s_{0} \mid P\right) \prod_{i=1}^{N} \prod_{k=1}^{N} p_{i k}^{N_{i k}(s)} p(P)
$$

The posterior density is demonstrated as follows

$$
p\left(\alpha_{i} \mid y, S\right) \infty \prod_{i=1}^{N} p\left(y_{i} \mid \alpha_{i}, y_{i-1}\right) p\left(\alpha_{i}\right)
$$

In this paper, prior distribution suggested was employed. The posterior distribution of $b$ is 
The Relation Between Growth, Energy Imports, Militarization and Current Account Balance in India, Brazil, Turkey and Pakistan

$$
p\left(b / y_{t}\right)=\pi\left(b_{0}\right) \pi\left(b_{+} / b_{0}\right)
$$

where $\mathrm{y}_{\mathrm{t}}$ denotes the data matrix up to time $\mathrm{T}$,

$$
\begin{aligned}
& \pi\left(b_{0}\right) \infty\left|B_{0}\right|^{T} \exp \left(-\frac{1}{2} \operatorname{trace}\left(B_{0} S B_{0}\right)\right) \\
& \pi\left(b_{+} \mid b_{0}\right)=\lambda\left((I \otimes U) b_{0} ; I \otimes V\right)
\end{aligned}
$$

$\lambda\left(\mu ; \sigma^{2}\right)$ means the normal density function with mean $\mu$ and variance $\sigma^{2} . S, U$ and $V$ are matrix functions in equations.

\section{Econometric Results}

\subsection{The Unit Root and Johansen test results}

The first differences of $y, m l, c$ and $c a$ were found as stationary as it can be seen from Table 1. We applied the point optimal test of Philips-Perron test, ElliottRothenberg-Stock Test and Ng and Perron test. Table 1 shows unit root test results. PP results show that the existence of a unit root cannot be rejected at the level for all countries. Then, Johansen test is employed to test the possibility of cointegration. If the null hypothesis is accepted, in this condition, the innovations of the variables, $d y$, $d m l, d c, d c a$, can be employed for MSBGC test.

Table 1. Unit root and Johansen test results for the analyzed countries

\begin{tabular}{|c|c|c|c|c|c|c|}
\hline & \multicolumn{7}{|c|}{} & $\begin{array}{l}\text { Elliott- } \\
\text { Rothenberg- } \\
\text { Stock Test } \\
\text { Statistic }\end{array}$ & $\begin{array}{c}\text { Philips } \\
\text { Perron Test }\end{array}$ \\
\hline \multicolumn{7}{|c|}{ Ng-Perron Test } \\
\hline $\mathrm{MZ}_{\mathrm{a}}$ & $\mathrm{MZ}_{\mathrm{t}}$ & MSB & MPT & & \\
\hline$y_{\mathrm{t}}$ & -2.01 & -1.38 & 0.67 & 8.97 & 28.13 & -5.256 \\
\hline$d y_{\mathrm{t}}$ & -24.59 & -3.44 & 0.11 & 1.05 & 1.132 & \\
\hline $\mathrm{ml}_{\mathrm{t}}$ & -1.96 & -1.22 & 0.74 & 7.16 & 7.88 & -4.03 \\
\hline$d m l_{\mathrm{t}}$ & -29.88 & -5.31 & 0.103 & 1.13 & 1.08 & \\
\hline$c_{t}$ & 0.18 & 0.35 & 0.93 & 11.44 & 8.22 & -3.07 \\
\hline$d c_{t}$ & -39.82 & -6.84 & 0.04 & 0.51 & 1.72 & \\
\hline$c a$ & -0.275 & -0.02 & 0.83 & 33.56 & 16.14 & -3.66 \\
\hline$d c a$ & -19.64 & -8.97 & 0.16 & 1.99 & 0.16 & \\
\hline \multicolumn{7}{|c|}{ Brazil } \\
\hline$y_{\mathrm{t}}$ & -2.78 & -1.08 & 0.97 & 8.97 & 16.73 & -4.52 \\
\hline$d y_{\mathrm{t}}$ & -24.59 & -9.74 & 0.19 & 1.15 & 1.32 & \\
\hline $\mathrm{ml}_{\mathrm{t}}$ & -3.76 & -1.27 & 0.34 & 7.16 & 8.18 & -4.01 \\
\hline$d m l_{\mathrm{t}}$ & -28.28 & -3.11 & 0.13 & 0.87 & 1.08 & \\
\hline$c_{t}$ & 0.38 & 0.85 & 0.93 & 19.44 & 8.22 & -3.13 \\
\hline
\end{tabular}


Melike Bildirici, Fazıl Kayıkçı

\begin{tabular}{|c|c|c|c|c|c|c|}
\hline$d c_{t}$ & -35.38 & -8.51 & 0.01 & 0.94 & 1.193 & \\
\hline $\mathrm{ca}$ & 0.38 & 0.55 & 0.99 & 11.44 & 12.22 & -3.13 \\
\hline$d c a$ & -31.38 & -4.85 & 0.101 & 0.20 & 1.093 & \\
\hline \multicolumn{6}{|c|}{ Turkey } & \\
\hline$y_{\mathrm{t}}$ & -1.63 & -1.77 & 0.69 & 9.72 & 4.19 & -7.46 \\
\hline$d y_{\mathrm{t}}$ & -29.01 & -5.97 & 0.18 & 0.28 & 0.48 & \\
\hline $\mathrm{ml}_{\mathrm{t}}$ & -2.04 & -1.19 & 0.76 & 8.29 & 9.06 & -3.55 \\
\hline$d m l_{\mathrm{t}}$ & -25.14 & -7.10 & 0.11 & 0.33 & 1.06 & \\
\hline$c_{t}$ & 0.35 & 0.12 & 0.81 & 11.39 & 17.08 & -3.94 \\
\hline$d c_{t}$ & -29.62 & -8.84 & 0.04 & 0.05 & 1.72 & \\
\hline $\mathrm{Ca}$ & -23.44 & -3.38 & 0.92 & 11.92 & 13.7 & -3.63 \\
\hline$d c a$ & -0.205 & \begin{tabular}{l|l}
-0.102 \\
\end{tabular} & 0.19 & 1.76 & 0.61 & \\
\hline \multicolumn{6}{|c|}{ Pakistan } & \\
\hline$y_{\mathrm{t}}$ & -3.12 & \begin{tabular}{|l|l}
-1.01 \\
\end{tabular} & 0.33 & 6.56 & 6.55 & -3.02 \\
\hline$d y_{\mathrm{t}}$ & -22.22 & -3.31 & 0.107 & 1.13 & 1.03 & \\
\hline $\mathrm{ml}_{\mathrm{t}}$ & -6.27 & -1.14 & 0.76 & 4.94 & 7.94 & -4.28 \\
\hline$d m l_{\mathrm{t}}$ & -23.34 & -4.88 & 0.12 & 1.35 & 1.27 & \\
\hline$c_{t}$ & -2.75 & -0.182 & 0.92 & 8.35 & 26.54 & -4.18 \\
\hline$d c_{t}$ & -20.24 & -4.27 & 0.26 & 1.313 & 0.56 & \\
\hline $\mathrm{Ca}$ & -1.81 & -1.38 & 0.97 & 8.718 & 18.83 & -3.77 \\
\hline$d c a$ & -26.59 & \begin{tabular}{l|l}
-4.74 \\
\end{tabular} & 0.11 & 1.037 & 1.13 & \\
\hline \multicolumn{6}{|c|}{ Johansen test results } & \\
\hline \multicolumn{2}{|c|}{ India } & Brazil & \multicolumn{2}{|c|}{\begin{tabular}{l|l} 
& Turkey
\end{tabular}} & Pakistan & $\begin{array}{l}\text { Critical } \\
\text { Values }\end{array}$ \\
\hline \multicolumn{2}{|c|}{$\mathrm{r}=0 \quad 38.62$} & $\mathrm{r}=0 \quad 41.48$ & \multicolumn{2}{|c|}{$\mathrm{r}=035.78$} & $\mathrm{r}=039.88$ & $\mathrm{r}=047.85$ \\
\hline \multicolumn{2}{|c|}{$\mathrm{r} \leq 20.42$} & $r \leq 22.68$ & \multicolumn{2}{|c|}{$\mathrm{r} \leq 113.92$} & $\mathrm{r} \leq 124.47$ & $r \leq 1 \quad 29.79$ \\
\hline \multicolumn{2}{|c|}{$\mathrm{r} \leq 213.02$} & $\mathrm{r} \leq 213.88$ & \multicolumn{2}{|c|}{$\mathrm{r} \leq 29.38$} & $\mathrm{r} \leq 211.52$ & $\mathrm{r} \leq 215.494$ \\
\hline \multicolumn{2}{|c|}{$\mathrm{r} \leq 31.1$} & $r \leq 31.0$ & \multicolumn{2}{|c|}{$r \leq 31.12$} & $r \leq 32.51$ & $r \leq 33.84$ \\
\hline
\end{tabular}

\subsection{MSBVAR Models and Dating}

In third stage, the selected models are MSIAH(3)BVAR(1) for Pakistan; MSIA(3)BVAR(2) for Brazil and MSIA(3)BVAR(1) for Turkey and India. The selection of models was based on the Akaike Information Criteria (AIC), Loglikelihood, LR test statistics. All models were chosen by depending upon the results of the diagnostic tests.

After selection of the models, business cycle dating of the selected models were compared with ECRI's, and OECD's business cycle datings. If these dates are similar ECRI dating, it will be accepted the proof of the accuracy of the model. The business cycle dating determined by the selected models and ECRI were given in 
The Relation Between Growth, Energy Imports, Militarization and Current Account Balance in India, Brazil, Turkey and Pakistan

Table 2 . The selected models detected the important recession years as the first enery crisis in 1974, second enery crisis in 1979, and the 2008 crisis as a whole.

Regime 3 represents high growth regime and/or low volatility regime, regime 2 represents moderate volatility and/or growth regime and regime 3 represents the crisis regime and/or high volatility regime.

In the selected MSBVAR models, the period of crisis regime, regime 1 has shorter time duration than the periods of economic growth, as regime 2 and regime 3. So the asymmetric behavior between economic growth and crisis periods was verified by depending upon the differences in length of time. According to these results, the transition probability matrix is ergodic and cannot be irreducible.

Table 2. Dating analysis (year: month)

\begin{tabular}{|c|c|c|c|}
\hline Turkey & India & Pakistan & Brazil \\
\hline $1977-1980$ & $1972-1973$ & $1973-1975$ & $1983-1987$ \\
\hline $\begin{array}{c}1984-1985: \\
1989-1989\end{array}$ & $1979-1980$ & $1986-1995$ & $1989-1992$ \\
\hline $1993-1994$ & $1991-1991$ & $2006-2011$ & $1997-1999$ \\
\hline $1998-1999$ & $1996-1997$ & & $2001-2003$ \\
\hline $2000-2001$ & & & $2003-2006$ \\
\hline $2008-2009$ & & & $2008-2009$ \\
\hline OECD [23] & ECRI [24] & & \\
\hline $1976: 09-1980: 07$ & $1972: 6-1973: 5$ & & \\
\hline $1981: 10-1982: 07$ & $1979: 4-1980: 3$ & & \\
\hline $1984: 06-1985: 08$ & $1991: 3-1991: 9$ & & \\
\hline $1987: 11-1989: 05$ & $1996: 5-1996: 11$ & & \\
\hline $1993: 08-1994: 07$ & & & \\
\hline $1998: 02-1999: 09$ & & & \\
\hline $2000: 08-2001: 12$ & & & \\
\hline $2008: 1-2009: 4$ & & & \\
\hline
\end{tabular}

The results for India were exhibited in Table 3. The posterior estimates were estimated as 911.32, 3791.09 and 1986.45 for regime 1, 2 and 3, respectively. The probabilities, $\operatorname{Prob}\left(\mathrm{s}_{\mathrm{t}}=1 \mid \mathrm{s}_{\mathrm{t}-1}=1\right)=0.63, \operatorname{Prob}\left(\mathrm{s}_{\mathrm{t}}=2 \mid \mathrm{s}_{\mathrm{t}-1}=2\right)=0.75$ and $\operatorname{Prob}\left(\mathrm{s}_{\mathrm{t}}=3 \mid \mathrm{s}_{\mathrm{t}-1}\right.$ $=3)=0.63$ show the persistence of regimes. It was determined that dominant regime is regime 2 and so showed the existence of asymmetry. 
Melike Bildirici, Fazıl Kayıkçı

Table 3. India, MSIA(3)-BVAR(1) model

\begin{tabular}{|c|c|c|c|c|c|c|c|c|c|c|c|c|}
\hline & \multicolumn{4}{|c|}{ Regime 1} & \multicolumn{4}{|c|}{ Regime 2} & \multicolumn{4}{|c|}{ Regime 3} \\
\hline & $d c a_{t}$ & $d c_{\mathrm{t}}$ & $d m l_{t}$ & dyt & $d c a_{t}$ & $d c_{t}$ & $d m l_{t}$ & dyt & $d c a_{t}$ & $d c_{t}$ & $d m l_{t}$ & dyt \\
\hline Constant & $\begin{array}{l}1.48 \\
(0.7)\end{array}$ & $\begin{array}{l}1.77 \\
(1.8)\end{array}$ & $\begin{array}{l}1.54 \\
(2.1)\end{array}$ & $\begin{array}{l}-2.06 \\
(-1.8)\end{array}$ & $\begin{array}{l}0.53 \\
(0.1) \\
\end{array}$ & $\begin{array}{l}1.82 \\
(6.1)\end{array}$ & $\begin{array}{l}1.13 \\
(7.7) \\
\end{array}$ & \begin{tabular}{|l}
2.07 \\
$(1.9)$
\end{tabular} & $\begin{array}{l}2.54 \\
(0.8) \\
\end{array}$ & $\begin{array}{l}1.19 \\
(3.8) \\
\end{array}$ & $\begin{array}{c}1.12 \\
(-3.8)\end{array}$ & $\begin{array}{l}1.73 \\
(1.2) \\
\end{array}$ \\
\hline$d c a_{t-1}$ & $\begin{array}{r}-0.05 \\
(-0.4) \\
\end{array}$ & $\begin{array}{l}0.01 \\
(0.5) \\
\end{array}$ & $\begin{array}{c}0.01 \\
(2.1) \\
\end{array}$ & $\begin{array}{r}-0.03 \\
(-2.2) \\
\end{array}$ & $\begin{array}{l}0.83 \\
(0.6) \\
\end{array}$ & $\begin{array}{c}-0.002 \\
(-2.1) \\
\end{array}$ & $\begin{array}{l}-0.00 \\
(-1.1) \\
\end{array}$ & $\begin{array}{l}-0.01 \\
-1.8 \\
\end{array}$ & \begin{tabular}{|l|}
0.87 \\
$(1.9)$ \\
\end{tabular} & $\begin{array}{l}-0.03 \\
(-3.1) \\
\end{array}$ & $\begin{array}{l}-0.02 \\
(-2.2) \\
\end{array}$ & $\begin{array}{l}-0.01 \\
(1.9) \\
\end{array}$ \\
\hline$d c_{t-1}$ & $\begin{array}{l}1.17 \\
(1.9)\end{array}$ & $\begin{array}{l}0.87 \\
(2.8)\end{array}$ & $\begin{array}{l}0.35 \\
(1.9)\end{array}$ & $\begin{array}{l}-3.76 \\
(-2.9)\end{array}$ & $\begin{array}{l}1.17 \\
(2.2)\end{array}$ & $\begin{array}{c}0.08 \\
(0.01)\end{array}$ & $\begin{array}{l}0.01 \\
(1.8)\end{array}$ & \begin{tabular}{|l}
-1.48 \\
$(-1.8$
\end{tabular} & $\begin{array}{l}-1.73 \\
(-1.1)\end{array}$ & $\begin{array}{l}0.01 \\
(2.2)\end{array}$ & \begin{tabular}{|l}
0.01 \\
$(1.9)$
\end{tabular} & $\begin{array}{l}1.58 \\
(2.2)\end{array}$ \\
\hline$d m l_{t-1}$ & $\begin{array}{c}1.08 \\
(2.1)\end{array}$ & $\begin{array}{r}1.46 \\
(2.1)\end{array}$ & $\begin{array}{r}-0.56 \\
(1.8)\end{array}$ & $\begin{array}{l}2.82 \\
(1.8) \\
\end{array}$ & $\begin{array}{l}1.28 \\
(1.7)\end{array}$ & $\begin{array}{l}0.03 \\
(1.8)\end{array}$ & $\begin{array}{l}0.01 \\
(1.8)\end{array}$ & $\begin{array}{l}-1.12 \\
(-1.9 \\
\end{array}$ & $\begin{array}{l}2.18 \\
(2.2)\end{array}$ & $\begin{array}{c}0.63 \\
(1.8) \\
\end{array}$ & $\begin{array}{l}-0.07 \\
(-2.3) \\
\end{array}$ & $\begin{array}{l}1.12 \\
(2.1)\end{array}$ \\
\hline$d y_{t-1}$ & $\begin{array}{l}0.03 \\
(0.3)\end{array}$ & $\begin{array}{l}-0.03 \\
(-1.1)\end{array}$ & $\begin{array}{c}-0.01 \\
(2.1)\end{array}$ & \begin{tabular}{|l|}
0.76 \\
$(1.7)$ \\
\end{tabular} & \begin{tabular}{|l}
0.45 \\
$(2.2)$
\end{tabular} & $\begin{array}{c}-0.01 \\
(-1.9)\end{array}$ & $\begin{array}{l}0.00 \\
(0.8)\end{array}$ & $\begin{array}{l}-0.58 \\
(-1.9 \\
\end{array}$ & $\begin{array}{l}1.42 \\
(5.7)\end{array}$ & \begin{tabular}{|c|}
0.05 \\
$(2.2)$
\end{tabular} & \begin{tabular}{|l}
0.001 \\
$(2.3)$
\end{tabular} & $\begin{array}{c}-0.57 \\
(1.8) \\
\end{array}$ \\
\hline \multicolumn{13}{|c|}{ Contemporaneous Correlation } \\
\hline & Prob. & \begin{tabular}{l|l}
. & $\begin{array}{l}\text { Po } \\
\text { es }\end{array}$ \\
\end{tabular} & $\begin{array}{l}\text { sterior } \\
\text { t. }\end{array}$ & & CA & C & MI & $\mathbf{L} \quad \mathbf{Y}$ & & & & \\
\hline Regime 1 & 0.177 & & 1.32 & $C A$ & 1 & & & & & $\mathbf{P}_{11}$ & & 63 \\
\hline Regime 2 & 0.635 & & 91.09 & C & 0.42 & 1 & & & & $\mathbf{P}_{22}$ & & 75 \\
\hline Regime 3 & 0.187 & & 86.45 & ML & -0.11 & -0.32 & 1 & 1 & & $\mathbf{P}_{33}$ & & 63 \\
\hline & & & & $\boldsymbol{Y}$ & -0.45 & -0.10 & & .3011 & & & & \\
\hline
\end{tabular}

Notes: t-statistics are given in parentheses. Log-likelihood:165.53 linear system:131.16 ; AIC: -4.16; linear system: -4.70 ; LR linearity: 68.75

In table 4, the MSIA(3)BVAR(2) model for Brazil showed that the moderate growth regime is most persistent regime and regime 1 follows moderate growth regime with 2.40 years on average. The high growth regime with 2.25 years is phase having the least duration. The posterior estimates were estimated as $881.77,3668.16$ and 1904.62 for regime 1,2 and 3 , respectively. $\operatorname{Prob}\left(\mathrm{s}_{\mathrm{t}}=1 \mid \mathrm{s}_{\mathrm{t}-1}=1\right)=0.88$, $\operatorname{Prob}\left(\mathrm{s}_{\mathrm{t}}=2 \mid \mathrm{s}_{\mathrm{t}-1}=2\right)=0.70$ and $\operatorname{Prob}\left(\mathrm{s}_{\mathrm{t}}=3 \mid \mathrm{s}_{\mathrm{t}-1}=3\right)=0.55$ reflect persistency of the regimes.

Table 4. Brazil, MSIA(3)-BVAR(2) model

\begin{tabular}{|c|c|c|c|c|c|c|c|c|c|c|c|c|}
\hline & \multicolumn{4}{|c|}{ Regime 1 } & \multicolumn{4}{c|}{ Regime 2 } & \multicolumn{4}{c|}{ Regime 3 } \\
\hline & $\boldsymbol{d c a}_{\mathbf{t}}$ & $\boldsymbol{d}_{\mathbf{t}}$ & $\boldsymbol{d m}_{\mathbf{t}}$ & $\mathbf{d y}_{\mathbf{t}}$ & $\boldsymbol{d c a}_{\mathbf{t}}$ & $\boldsymbol{d}_{\mathbf{t}}$ & $\boldsymbol{d m}_{\mathbf{t}}$ & $\mathbf{d y}_{\mathbf{t}}$ & $\boldsymbol{d c a}_{\mathbf{t}}$ & $\boldsymbol{d}_{\mathbf{t}}$ & $\boldsymbol{d m I}_{\mathbf{t}}$ & $\mathbf{d y}_{\mathbf{t}}$ \\
\hline Const & 2.15 & -2.02 & -4.92 & 0.37 & -8.1 & 7.50 & 3.26 & 0.86 & 1.92 & 2.9 & 1.43 & 0.11 \\
ant & $(2.2)$ & $(-2.3)$ & $(-2.4)$ & $(1.24)$ & $(-1.5)$ & $(2.2)$ & $(0.8)$ & $(13.4)$ & $(0.5)$ & $(2.5)$ & $(5.5)$ & $(0.4)$ \\
\hline $\boldsymbol{d c a}_{\mathbf{t}-\mathbf{1}}$ & -0.77 & -0.29 & -0.06 & 0.02 & -0.22 & -0.19 & -0.09 & -0.04 & 0.06 & 0.45 & 1.32 & 0.0003 \\
& $(-7.6)$ & $(-4.4)$ & $(-2.6)$ & $(12.4)$ & $(2.2)$ & $(3.7)$ & $(0.8)$ & $(-2)$ & $(0.7)$ & $(2.8)$ & $(8.16)$ & $(0.2)$ \\
\hline $\boldsymbol{d c a}_{\mathbf{t}-\mathbf{2}}$ & -1.7 & 0.72 & 0.43 & -0.02 & -0.06 & -0.17 & -0.02 & -0.02 & -1.17 & -0.92 & -0.41 & -0.002 \\
& $(-6.3)$ & $(4.3)$ & $(2.2)$ & $(-6.2)$ & $(-0.1)$ & $(-2.5)$ & $(1.9)$ & $(-3.4)$ & $(-2.1)$ & $(-3.7)$ & $(-1.1)$ & $(0.4)$ \\
\hline $\boldsymbol{d c}_{\boldsymbol{t}-\mathbf{1}}$ & 1.38 & 5.11 & 0.12 & -0.09 & -0.06 & -0.07 & 0.02 & 0.02 & -0.68 & 1.14 & -0.15 & 0.0006 \\
& $(2.4)$ & $(14.1)$ & $(0.3)$ & $(-13)$ & $(1.9)$ & $(-1.2)$ & $(1.8)$ & $(2.4)$ & $(-2.6)$ & $(5.1)$ & $(-3.7)$ & $(0.5)$ \\
\hline $\boldsymbol{d c}_{\boldsymbol{t}-\mathbf{2}}$ & 0.36 & 0.43 & -0.3 & -0.02 & -0.04 & -0.07 & 0.18 & -0.04 & 1.98 & 1.27 & -0.33 & 0.021 \\
& $(3.1)$ & $(5.7)$ & $(-3.5)$ & $(-11)$ & $(-1.1)$ & $(-3.4)$ & $(2.3)$ & $(-3)$ & $(6.4)$ & $(3.9)$ & $(-3.1)$ & $(3.4)$ \\
\hline
\end{tabular}


The Relation Between Growth, Energy Imports, Militarization and Current Account Balance in India, Brazil, Turkey and Pakistan

\begin{tabular}{|c|c|c|c|c|c|c|c|c|c|c|c|c|}
\hline$d m I_{t-1}$ & $\begin{array}{l}13.2 \\
(1.1)\end{array}$ & $\begin{array}{l}2.81 \\
(3.2) \\
\end{array}$ & $\begin{array}{l}-9.27 \\
(2.7)\end{array}$ & $\begin{array}{r}1.91 \\
(12.2\end{array}$ & $\begin{array}{l}9.15 \\
(2.2) \\
\end{array}$ & $\begin{array}{c}1.2 \\
(1.6)\end{array}$ & $\begin{array}{l}4.11 \\
(1.7) \\
\end{array}$ & $\begin{array}{l}0.21 \\
(2.9)\end{array}$ & $\begin{array}{l}3.81 \\
(2.9)\end{array}$ & $\begin{array}{l}1.19 \\
(3.2) \\
\end{array}$ & $\begin{array}{c}-2.1 \\
(-5.7) \\
\end{array}$ & \begin{tabular}{|l}
0.89 \\
$(2.7)$ \\
\end{tabular} \\
\hline$d m l_{t-2}$ & $\begin{array}{l}2.11 \\
(7.9) \\
\end{array}$ & $\begin{array}{l}1.42 \\
\text { (5.5) }\end{array}$ & $\begin{array}{c}5.7 \\
(2.3) \\
\end{array}$ & $\begin{array}{l}-1.32 \\
(-4.1)\end{array}$ & \begin{tabular}{|l|l}
2 & 1.53 \\
) & $(2.4)$ \\
\end{tabular} & $\begin{array}{l}6.21 \\
(1.8)\end{array}$ & $\begin{array}{c}-6.1 \\
(-0.6)\end{array}$ & $\begin{array}{l}-0.04 \\
(0.5)\end{array}$ & $\begin{array}{c}1.55 \\
(1.66) \\
\end{array}$ & $\begin{array}{l}1.74 \\
(3.6) \\
\end{array}$ & \begin{tabular}{|l|}
1.74 \\
$(3.1)$
\end{tabular} & $\begin{array}{l}-0.02 \\
(-0.3) \\
\end{array}$ \\
\hline$d y_{t-1}$ & $\begin{array}{l}-0.79 \\
(2.7)\end{array}$ & $\begin{array}{l}0.27 \\
(1.8)\end{array}$ & $\begin{array}{l}-0.11 \\
(-0.1)\end{array}$ & $\begin{array}{r}0.035 \\
(4.5)\end{array}$ & \begin{tabular}{l|l}
5 & 0.02 \\
& $(0.6)$
\end{tabular} & $\begin{array}{l}0.08 \\
(0.8)\end{array}$ & $\begin{array}{l}0.08 \\
(1.2)\end{array}$ & $\begin{array}{l}0.03 \\
(4.3)\end{array}$ & $\begin{array}{l}-0.25 \\
(-0.1)\end{array}$ & $\begin{array}{l}-0.20 \\
(-3.1)\end{array}$ & $\begin{array}{l}-1.15 \\
(-5.3)\end{array}$ & $\begin{array}{l}0.002 \\
(0.5)\end{array}$ \\
\hline$d y_{t-2}$ & $\begin{array}{r}-2.87 \\
(-3) \\
\end{array}$ & $\begin{array}{l}0.04 \\
(0.2)\end{array}$ & $\begin{array}{l}-0.17 \\
(-0.7)\end{array}$ & $\begin{array}{r}0.04 \\
(12.5 \\
\end{array}$ & \begin{tabular}{|l|l}
4 & -0.06 \\
$5)$ & $(-1.1)$ \\
\end{tabular} & \begin{tabular}{|l}
-0.02 \\
$(-0.1)$ \\
\end{tabular} & \begin{tabular}{|l|}
-0.09 \\
$(-1.3)$ \\
\end{tabular} & $\begin{array}{c}-0.02 \\
(-2)\end{array}$ & $\begin{array}{l}-0.10 \\
(-1.4)\end{array}$ & $\begin{array}{l}0.09 \\
(1.1) \\
\end{array}$ & $\begin{array}{l}-0.58 \\
(-8.1) \\
\end{array}$ & $\begin{array}{l}0.002 \\
(1.7)\end{array}$ \\
\hline \multicolumn{13}{|c|}{ Contemporaneous Correlation } \\
\hline \multicolumn{4}{|c|}{\begin{tabular}{|l|l|} 
Prob. & $\begin{array}{l}\text { Posteri } \\
\text { or est. }\end{array}$
\end{tabular}} & \multicolumn{2}{|c|}{ CA } & $\mathbf{C}$ & ML & $\mathbf{Y}$ & & & & \\
\hline \multirow{2}{*}{\multicolumn{2}{|c|}{\begin{tabular}{|l|} 
Regime 1 \\
Regime 2 \\
\end{tabular}}} & \multicolumn{2}{|c|}{0.1845881 .77} & $\overline{C A}$ & 1 & & & & & \multicolumn{2}{|c|}{$\mathbf{P}_{11}$} & 0.88 \\
\hline & & \multirow{3}{*}{$|0.594|$} & 3668.16 & $C$ & \begin{tabular}{|l|}
-0.38 \\
\end{tabular} & 1 & & & & \multicolumn{2}{|c|}{$\mathbf{P}_{22}$} & 0.70 \\
\hline \multirow{2}{*}{\multicolumn{2}{|c|}{ Regime 3}} & & 04.62 & ML & -0.20 & -0.41 & 11 & & & \multicolumn{2}{|c|}{$\mathbf{P}_{33}$} & 0.55 \\
\hline & & & & $\bar{Y}$ & 0.36 & $\mid-0.30$ & 0.09 & 1 & & & & \\
\hline
\end{tabular}

Notes: t-statistics are given in parentheses. log-likelihood:85.27; linear system:93.87; AIC:1.80; LR linearity test:358.29

In table 5, in the MSIA(3)BVAR(1) model for Turkey we found that the most persistent regime was regime 2 . The results showed that there is a low chance that a crisis is followed by a period of high growth. The posterior estimates were estimated as 593.66, 2469.62 and 1282.31 for regime 1, 2 and 3, respectively. In the regime 1 , the probability of turning to the regime 2 is $0.1065(10.65 \%)$ but staying in the crisis stage is more possible with probability of $0.71(71 \%)$.

Table 5. Turkey, MSIA(3)-BVAR(1) model

\begin{tabular}{|c|c|c|c|c|c|c|c|c|c|c|c|c|}
\hline & \multicolumn{4}{|c|}{ Regime 1} & \multicolumn{4}{|c|}{ Regime 2} & \multicolumn{4}{|c|}{ Regime 3} \\
\hline & $d_{c} a_{t}$ & $d c_{\mathrm{t}}$ & $d m l_{t}$ & $\mathbf{d y}_{\mathrm{t}}$ & $d_{c a} a_{t}$ & $d c_{\mathrm{t}}$ & $d m l_{t}$ & $d y_{t}$ & $d^{d c a_{\mathrm{t}}}$ & $d c_{\mathrm{t}}$ & $d m l_{t}$ & $\mathbf{d y}_{\mathrm{t}}$ \\
\hline Constant & \begin{tabular}{|l|}
3.51 \\
$(1.7)$
\end{tabular} & $\begin{array}{l}-0.16 \\
(1.4)\end{array}$ & $\begin{array}{l}-1.41 \\
(2.05)\end{array}$ & $\begin{array}{l}0.53 \\
(2.6)\end{array}$ & $\begin{array}{c}1.87 \\
(1.7)\end{array}$ & $\begin{array}{l}0.88 \\
(2.2)\end{array}$ & $\begin{array}{c}2.58 \\
(4.05)\end{array}$ & $\begin{array}{l}0.61 \\
(5.2)\end{array}$ & $\begin{array}{l}1.83 \\
(1.3)\end{array}$ & $\begin{array}{l}0.83 \\
(1.8)\end{array}$ & $\begin{array}{l}-1.52 \\
(-3.8)\end{array}$ & $\begin{array}{l}0.63 \\
(3.9)\end{array}$ \\
\hline $\operatorname{dca}_{t-1}$ & $\begin{array}{c}0.68 \\
(0.4)\end{array}$ & $\begin{array}{l}0.03 \\
(0.3)\end{array}$ & $\begin{array}{l}-0.09 \\
(-1.4)\end{array}$ & $\begin{array}{l}-0.06 \\
(3.9)\end{array}$ & $\begin{array}{l}-0.15 \\
(-0.7) \\
\end{array}$ & $\begin{array}{l}0.13 \\
(1.8) \\
\end{array}$ & $\begin{array}{l}-0.24 \\
(-1.9)\end{array}$ & $\begin{array}{l}-0.09 \\
(-2.9)\end{array}$ & \begin{tabular}{|l|}
0.38 \\
$(1.9)$ \\
\end{tabular} & $\begin{array}{l}0.17 \\
(1.8)\end{array}$ & \begin{tabular}{|c|}
-0.002 \\
$(-2.3)$
\end{tabular} & $\begin{array}{c}-0.002 \\
(-0.8)\end{array}$ \\
\hline$d c_{t-1}$ & $\begin{array}{c}4.78 \\
(1.9)\end{array}$ & $\begin{array}{l}0.20 \\
(0.7) \\
\end{array}$ & $\begin{array}{l}-0.43 \\
(-0.1)\end{array}$ & $\begin{array}{c}0.02 \\
5(2.2)\end{array}$ & \begin{tabular}{|l|}
5.13 \\
$(1.8)$ \\
\end{tabular} & $\begin{array}{c}0.42 \\
(1.8) \\
\end{array}$ & $\begin{array}{l}-3.15 \\
(-1.9)\end{array}$ & $\begin{array}{l}-0.22 \\
(-2.9) \\
\end{array}$ & $\begin{array}{l}-3.28 \\
(-1.8) \\
\end{array}$ & $\begin{array}{l}-0.42 \\
(-1.6)\end{array}$ & $\begin{array}{l}-0.36 \\
(-3.6) \\
\end{array}$ & $\begin{array}{l}-0.05 \\
(-0.8) \\
\end{array}$ \\
\hline$d m l_{t-1}$ & $\begin{array}{c}0.04 \\
(1.8)\end{array}$ & $\begin{array}{l}0.03 \\
(1.8)\end{array}$ & $\begin{array}{l}-1.31 \\
(-5.4)\end{array}$ & $\begin{array}{c}0.026 \\
(0.5) \\
\end{array}$ & $\begin{array}{c}0.31 \\
(2.3) \\
\end{array}$ & $\begin{array}{c}0.03 \\
(1.9) \\
\end{array}$ & $\begin{array}{l}-0.53 \\
(-2.4)\end{array}$ & $\begin{array}{l}0.04 \\
(8.4)\end{array}$ & \begin{tabular}{|l|}
1.14 \\
$(2.8)$ \\
\end{tabular} & $\begin{array}{l}0.19 \\
(1.8) \\
\end{array}$ & \begin{tabular}{|l|}
-0.34 \\
$(-0.9)$ \\
\end{tabular} & $\begin{array}{l}-0.01 \\
(-2.9) \\
\end{array}$ \\
\hline$d y_{t-1}$ & $\begin{array}{l}2.77 \\
(1.5)\end{array}$ & $\begin{array}{c}0.9 \\
(1.8)\end{array}$ & $\begin{array}{l}4.86 \\
(2.5)\end{array}$ & $\begin{array}{l}0.51 \\
(1.8)\end{array}$ & \begin{tabular}{|l|}
-2.82 \\
$(-2.3)$
\end{tabular} & $\begin{array}{l}-0.42 \\
(-0.9)\end{array}$ & $\begin{array}{l}1.99 \\
(3.5)\end{array}$ & $\begin{array}{l}0.68 \\
(6.8)\end{array}$ & $\begin{array}{l}-4.58 \\
(-1.8)\end{array}$ & $\begin{array}{l}0.68 \\
(1.9)\end{array}$ & \begin{tabular}{|l|}
1.41 \\
$(2.3)$
\end{tabular} & $\begin{array}{l}0.56 \\
(3.4)\end{array}$ \\
\hline \multicolumn{13}{|c|}{ Contemporaneous Correlation } \\
\hline & Prob & \multicolumn{2}{|c|}{$\begin{array}{l}\text { Posterior } \\
\text { est. }\end{array}$} & & CA & $\mathbf{C}$ & ML & $\mathbf{Y}$ & & & & \\
\hline Regime 1 & 0.301 & \multicolumn{2}{|c|}{593.66} & $C A$ & 1 & & & & & $\mathbf{P}_{11}$ & \multicolumn{2}{|c|}{0.71} \\
\hline Regime 2 & 0.377 & \multicolumn{2}{|c|}{2469.62} & $C$ & 0.52 & 1 & & & & $\mathbf{P}_{22}$ & \multicolumn{2}{|c|}{0.72} \\
\hline
\end{tabular}


Melike Bildirici, Fazıl Kayıkçı

\begin{tabular}{|l|l|l|l|l|l|l|l|l|l|l|}
\hline Regime 3 & 0.302 & 1282.31 & $\boldsymbol{M L}$ & -0.45 & -0.21 & 1 & & & $\mathbf{P}_{\mathbf{3}}$ & 0.61 \\
\hline & & & $\boldsymbol{Y}$ & -0.65 & -0.16 & 0.22 & 1 & & & \\
\hline
\end{tabular}

Notes:t-statistics are given in parentheses. Log-likelihood=184.37 linear system:123.52 AIC: -5.5577; LR linearity:121.72;

MSIAH(3)BVAR(1) model was selected for Pakistan and the results were given in Table 6 . The most persistent regime with 3.16 years is determined as regime 2. It was found as $\operatorname{Prob}\left(\mathrm{s}_{\mathrm{t}}=1 \mid \mathrm{s}_{\mathrm{t}-1}=1\right)=0.61, \operatorname{Prob}\left(\mathrm{s}_{\mathrm{t}}=2 \mid \mathrm{s}_{\mathrm{t}-1}=2\right)=0.68$ and $\operatorname{Prob}\left(\mathrm{s}_{\mathrm{t}}=3 \mid \mathrm{s}_{\mathrm{t}-1}\right.$ $=3)=0.64$ and it was accepted the persistence of regimes and the important asymmetries with $\mathrm{p}_{11}=0.24, \mathrm{p}_{22}=0.45$ and $\mathrm{p}_{33}=0.31$.

Table 6. Pakistan, MSIAH(3)-BVAR(1) model

\begin{tabular}{|c|c|c|c|c|c|c|c|c|c|c|c|c|}
\hline & \multicolumn{4}{|c|}{ Regime 1} & \multicolumn{4}{|c|}{ Regime 2} & \multicolumn{4}{|c|}{ Regime 3} \\
\hline & $d c a_{t}$ & $d c_{\mathrm{t}}$ & $d m I_{t}$ & $d_{t}$ & $d c a_{t}$ & $d c_{\mathrm{t}}$ & $d m I_{t}$ & dyt & $d c a_{\mathrm{t}}$ & $d c_{\mathrm{t}}$ & $d m I_{t}$ & $d y_{t}$ \\
\hline Constant & $\begin{array}{l}-0.04 \\
(-5.1)\end{array}$ & \begin{tabular}{|c|}
0.13 \\
$(2.8)$
\end{tabular} & $\begin{array}{c}0.00003 \\
(0.1)\end{array}$ & \begin{tabular}{|c|}
-0.78 \\
$(0.1)$
\end{tabular} & $\begin{array}{c}-1.63 \\
(-1)\end{array}$ & $\begin{array}{c}0.93 \\
(1.3)\end{array}$ & $\begin{array}{l}0.77 \\
(0.3)\end{array}$ & $\begin{array}{l}1.83 \\
(0.3)\end{array}$ & \begin{tabular}{|c|}
1.73 \\
$(0.46)$
\end{tabular} & $\begin{array}{l}1.21 \\
(-2)\end{array}$ & $\begin{array}{l}1.62 \\
(4.1)\end{array}$ & $\begin{array}{l}-2.27 \\
(-1.3)\end{array}$ \\
\hline$d c a_{t-1}$ & $\begin{array}{l}1.88 \\
(2.6)\end{array}$ & $\begin{array}{l}-0.05 \\
(2.9)\end{array}$ & $\begin{array}{c}-0.003 \\
(-0.3)\end{array}$ & $\begin{array}{c}0.65 \\
(0.1)\end{array}$ & $\begin{array}{l}-0.12 \\
(-2.8)\end{array}$ & $\begin{array}{l}0.01 \\
(1.8) \\
\end{array}$ & $\begin{array}{l}0.02 \\
(2.8) \\
\end{array}$ & $\begin{array}{c}0.08 \\
(1)\end{array}$ & \begin{tabular}{|l|}
-0.77 \\
$(-1.6)$ \\
\end{tabular} & $\begin{array}{l}0.01 \\
(2.3) \\
\end{array}$ & $\begin{array}{l}-0.01 \\
(-0.3)\end{array}$ & $\begin{array}{l}0.03 \\
(1.9)\end{array}$ \\
\hline$d c_{t-1}$ & $\begin{array}{l}2.62 \\
(1.9)\end{array}$ & $\begin{array}{l}0.50 \\
(2.5)\end{array}$ & $\begin{array}{c}-0.002 \\
(-0.1)\end{array}$ & $\begin{array}{l}0.92 \\
(0.5)\end{array}$ & \begin{tabular}{|l|}
3.74 \\
$(2.6)$
\end{tabular} & $\begin{array}{c}-0.38 \\
(2.5)\end{array}$ & $\begin{array}{l}0.06 \\
(1.8)\end{array}$ & $\begin{array}{c}-1.54 \\
(1.9)\end{array}$ & \begin{tabular}{|c|}
1.86 \\
$(1.8)$ \\
\end{tabular} & $\begin{array}{c}0.05 \\
(2.1)\end{array}$ & $\begin{array}{l}0.72 \\
(3.7)\end{array}$ & $\begin{array}{l}1.28 \\
(2.2)\end{array}$ \\
\hline$d m l_{t-1}$ & $\begin{array}{l}3.07 \\
\text { (3.3) }\end{array}$ & $\begin{array}{c}0.24 \\
(1.8)\end{array}$ & $\begin{array}{l}0.26 \\
(1.2)\end{array}$ & $\begin{array}{c}-0.31 \\
(-3.2)\end{array}$ & $\begin{array}{l}2.92 \\
(3.7)\end{array}$ & $\begin{array}{l}0.001 \\
(2.5)\end{array}$ & $\begin{array}{l}0.03 \\
(3.5)\end{array}$ & $\begin{array}{l}-2.08 \\
(2.1)\end{array}$ & \begin{tabular}{|l|}
2.19 \\
$(2.2)$ \\
\end{tabular} & $\begin{array}{l}0.09 \\
(2.2)\end{array}$ & $\begin{array}{l}0.06 \\
(0.8)\end{array}$ & $\begin{array}{l}-3.86 \\
(-3.2)\end{array}$ \\
\hline$d y_{t-1}$ & $\begin{array}{l}1.82 \\
(2.2)\end{array}$ & \begin{tabular}{|l|}
0.03 \\
$(1.8)$ \\
\end{tabular} & $\begin{array}{l}0.15 \\
(2.9) \\
\end{array}$ & $\begin{array}{c}0.145 \\
(0.8) \\
\end{array}$ & \begin{tabular}{|l}
0.15 \\
$(1.9)$ \\
\end{tabular} & $\begin{array}{l}0.04 \\
(1.7)\end{array}$ & $\begin{array}{c}0.0003 \\
(0.1)\end{array}$ & \begin{tabular}{|c|}
-0.14 \\
$(0.9)$
\end{tabular} & \begin{tabular}{|l|}
-1.06 \\
$(-2.1)$ \\
\end{tabular} & $\begin{array}{l}0.001 \\
(1.9)\end{array}$ & $\begin{array}{l}-0.01 \\
(-1.5)\end{array}$ & \begin{tabular}{|l|}
0.23 \\
$(3.4)$
\end{tabular} \\
\hline
\end{tabular}

\section{Contemporaneous Correlation}

\begin{tabular}{|l|l|l|l|l|l|l|l|l|l|l|}
\hline & Prob. & $\begin{array}{l}\text { Posterior } \\
\text { est. }\end{array}$ & & CA & C & ML & Y & & & \\
\hline Regime 1 & 0.227 & 716.41 & CA & 1 & & & & & $\mathbf{P}_{\mathbf{1 1}}$ & 0.81 \\
\hline Regime 2 & 0.6012 & 2980.26 & $\mathbf{C}$ & 0.16 & 1 & & & & $\mathbf{P}_{\mathbf{2 2}}$ & 0.68 \\
\hline Regime 3 & 0.1716 & 1547.44 & $\mathbf{M L}$ & -0.18 & 0.41 & 1 & & & $\mathbf{P}_{\mathbf{3 3}}$ & 0.64 \\
\hline & & & $\mathbf{Y}$ & 0.42 & -0.35 & 0.41 & 1 & & & \\
\hline
\end{tabular}

Notes: t-statistics are given in parentheses. log-likelihood: 242.4697 linear system:161.8261; AIC: -6.6577; LR linearity test:161.28.

\subsection{Causality results}

For analysed countries, causality results determined by any regime were summarised in the Table 7. According to the results for India, bi-directional Bayesian causal nexus between militarization and energy imports in all regimes was found, between militarization and current account, and between real GDP and militarization in regime 1 and 3 and between real GDP and energy imports, between real GDP and current account in regime 2 and 3 and between energy imports and current account in regime 2. 
The Relation Between Growth, Energy Imports, Militarization and Current Account Balance in India, Brazil, Turkey and Pakistan

Table 7. Summary of the Granger causality test results.

\begin{tabular}{|c|c|c|c|}
\hline Country & Regime1 & Regime2 & Regime3 \\
\hline \multirow{6}{*}{ INDIA } & $m l \leftrightarrow c$ & $m l \leftrightarrow c$ & $m l \leftrightarrow c$ \\
\hline & $y \leftrightarrow m l$ & $m l \rightarrow y$ & $m l \leftrightarrow y$ \\
\hline & $m l \leftrightarrow c a$ & $m l \rightarrow c a$ & $m l \leftrightarrow c a$ \\
\hline & $c a \rightarrow y$ & $c a \leftrightarrow y$ & $y \leftrightarrow c a$ \\
\hline & $c \rightarrow c a$ & $c \leftrightarrow c a$ & $c a \rightarrow c$ \\
\hline & $c \rightarrow y$ & $y \leftrightarrow c$ & $y \leftrightarrow c$ \\
\hline \multirow{6}{*}{ BRAZIL } & $m l \leftrightarrow c$ & $m l \leftrightarrow c$ & $m l \leftrightarrow c$ \\
\hline & $m l \rightarrow y$ & $m l \rightarrow y$ & $m l \leftrightarrow y$ \\
\hline & $m l \leftrightarrow c a$ & $m l \leftrightarrow c a$ & $m l \leftrightarrow c a$ \\
\hline & $y \leftrightarrow c a$ & $c a \rightarrow y$ & $y \neq c a$ \\
\hline & $c \leftrightarrow c a$ & $c \leftrightarrow c a$ & $c \leftrightarrow c a$ \\
\hline & $y \leftrightarrow c$ & $c \rightarrow y$ & $y \leftrightarrow c$ \\
\hline \multirow{6}{*}{ TURKEY } & $m l \rightarrow c$ & $m l \leftrightarrow c$ & $m l \leftrightarrow c$ \\
\hline & $y \rightarrow m l$ & $m l \leftrightarrow y$ & $m l \leftrightarrow y$ \\
\hline & $m l \rightarrow c a$ & $m l \leftrightarrow c a$ & $m l \leftrightarrow c a$ \\
\hline & $c a \rightarrow y$ & $c a \leftrightarrow y$ & $y \rightarrow c a$ \\
\hline & $c \rightarrow c a$ & $c \leftrightarrow c a$ & $c \leftrightarrow c a$ \\
\hline & $y \leftrightarrow c$ & $c \rightarrow y$ & $y \rightarrow c$ \\
\hline \multirow{6}{*}{ PAKISTAN } & $m l \rightarrow c$ & $m l \leftrightarrow c$ & $m l \leftrightarrow C$ \\
\hline & $y \leftrightarrow m l$ & $m l \rightarrow y$ & $m l \rightarrow y$ \\
\hline & $m l \rightarrow c a$ & $m l \leftrightarrow c a$ & $m l \rightarrow c a$ \\
\hline & $y \rightarrow c a$ & $y \rightarrow c a$ & $y \leftrightarrow c a$ \\
\hline & $c \leftrightarrow c a$ & $c \leftrightarrow c a$ & $c \leftrightarrow c a$ \\
\hline & $y \rightarrow c$ & $y \leftrightarrow c$ & $y \leftrightarrow c$ \\
\hline
\end{tabular}

The model for the Brazil show two-way Bayesian causal nexus between militarization and energy imports, and between militarization and current account, and between energy imports and current account in all regimes, and between real GDP and energy imports in regime 1 and 3 and there is the evidence of none causality between real GDP and current account in regime 3 but two-way causal nexus between real GDP and current account in regime 1 .

For Turkey, there is bidirectional Bayesian Granger causal nexus between energy imports and economic growth in regime 1 but unidirectional causality from energy imports to real GDP in regime 2 and from economic growth to energy imports in regime 3. There is the evidence of bi-directional Bayesian causal nexus between economic growth and militarization, between current account and militarization, between energy imports and current account in regime 2 and 3. There is one-way 
Melike Bildirici, Fazıl Kayıkçı

Bayesian causality from real GDP to militarization, from militarization to current account and from energy imports to current account in regime 1.

The selected model for Pakistan shows the evidence for two-way Bayesian Granger causal nexus between current account and energy imports in all regimes, and between militarization and energy imports in regime 2 and 3 . There is the evidence of one-way Bayesian causal nexus from real GDP to current account in regime 1 and 2, and from real GDP to energy imports and from militarization to energy imports in regime 1.

\subsection{Traditional linear Granger causality test results}

The causality results are very important to determine the economic policies since they can produce wrong economic policies if the stages of business cycle are disregarded. The results obtained by traditional method will be compared with the MSBGC test. The important differences between methods were found in Table 8.

Table 8. Traditional Granger causality test results

\begin{tabular}{|c|c|c|c|c|c|}
\hline Countries & Direction & Statistics & Probability & Decision & \\
\hline \multirow{12}{*}{ INDIA } & $\Delta l m l \rightarrow \Delta l y$ & 1.41016 & 0.2419 & No & \multirow[t]{2}{*}{$\mathrm{ml} \neq \mathrm{y}$} \\
\hline & $\Delta l y \rightarrow \Delta \operatorname{lml}$ & 0.97839 & 0.3284 & No & \\
\hline & $\Delta l c a \rightarrow \Delta l y$ & 0.04272 & 0.8373 & No & \multirow[t]{2}{*}{$\mathrm{ca} \neq \mathrm{y}$} \\
\hline & $\Delta l y \rightarrow \Delta l c a$ & 0.17537 & 0.6776 & No & \\
\hline & $\Delta l m l \rightarrow \Delta l c a$ & 2.44415 & 0.1258 & No & \multirow[t]{2}{*}{$\mathrm{ml} \neq \mathrm{ca}$} \\
\hline & $\Delta l c a \rightarrow \Delta l m l$ & 0.34563 & 0.5599 & No & \\
\hline & $\Delta l m l \rightarrow \Delta l c$ & 0.84185 & 0.3632 & No & \multirow[t]{2}{*}{$\mathrm{ml} \neq \mathrm{C}$} \\
\hline & $\Delta l c \rightarrow \Delta \operatorname{lml}$ & 0.17995 & 0.6732 & No & \\
\hline & $\Delta l c \rightarrow \Delta l y$ & 0.39854 & 0.5313 & No & \multirow[t]{2}{*}{$\mathrm{C} \neq \mathrm{y}$} \\
\hline & $\Delta l y \rightarrow \Delta l c$ & 0.07365 & 0.7874 & No & \\
\hline & $\Delta l c \rightarrow \Delta l c a$ & 1.69015 & 0.2010 & No & \multirow[t]{2}{*}{$\mathrm{ca} \neq \mathrm{C}$} \\
\hline & $\Delta l c a \rightarrow \Delta l c$ & 1.47894 & 0.2311 & No & \\
\hline \multirow{10}{*}{ BRAZIL } & $\Delta I m l \rightarrow \Delta l y$ & 0.03025 & 0.8626 & No & \multirow[t]{2}{*}{$\mathrm{ml} \neq \mathrm{y}$} \\
\hline & $\Delta l y \rightarrow \Delta \operatorname{lml}$ & 0.37405 & 0.5435 & No & \\
\hline & $\Delta l c a \rightarrow \Delta l y$ & 2.6878 & 0.1088 & Yes & \multirow[t]{2}{*}{$\mathrm{ca} \rightarrow \mathrm{y}$} \\
\hline & $\Delta l y \rightarrow \Delta l c a$ & 0.10635 & 0.7460 & No & \\
\hline & $\Delta l m l \rightarrow \Delta l c a$ & 0.07014 & 0.7925 & No & \multirow[t]{2}{*}{$\mathrm{ml} \neq \mathrm{ca}$} \\
\hline & $\Delta l c a \rightarrow \Delta l m l$ & 0.37570 & 0.5433 & No & \\
\hline & $\Delta l m l \rightarrow \Delta l c$ & 0.09773 & 0.7558 & No & \multirow[t]{2}{*}{$\mathrm{ml}_{\neq} \mathrm{C}$} \\
\hline & $\Delta l c \rightarrow \Delta \operatorname{lml}$ & 0.09222 & 0.7626 & No & \\
\hline & $\Delta l c \rightarrow \Delta l y$ & 0.21060 & 0.6482 & No & \multirow[t]{2}{*}{$\mathrm{y} \rightarrow \mathrm{c}$} \\
\hline & $\Delta l y \rightarrow \Delta l c$ & 3.18673 & 0.0802 & Yes & \\
\hline
\end{tabular}


The Relation Between Growth, Energy Imports, Militarization and Current Account Balance in India, Brazil, Turkey and Pakistan

\begin{tabular}{|c|c|c|c|c|c|}
\hline & $\Delta l c \rightarrow \Delta l c a$ & 0.01918 & 0.8905 & No & \multirow[t]{2}{*}{$\mathrm{ca} \rightarrow \mathrm{c}$} \\
\hline & $\Delta l c a \rightarrow \Delta l c$ & 10.2019 & 0.0027 & Yes & \\
\hline \multirow{12}{*}{ TURKEY } & $\Delta l m l \rightarrow \Delta l y$ & 1.43 & 0.238 & No & \multirow[t]{2}{*}{$\mathrm{ml} \neq \mathrm{y}$} \\
\hline & $\Delta l y \rightarrow \Delta \operatorname{lml}$ & 2.064 & 0.1868 & No & \\
\hline & $\Delta l c a \rightarrow \Delta l y$ & 0.0709 & 0.791 & No & \multirow[t]{2}{*}{$\mathrm{ca} \neq \mathrm{y}$} \\
\hline & $\Delta l y \rightarrow \Delta l c a$ & $2.4 \mathrm{E}-06$ & 0.9988 & No & \\
\hline & $\Delta l m l \rightarrow \Delta l c a$ & 0.26837 & 0.6076 & No & \multirow[t]{2}{*}{$\mathrm{ml}_{\neq} \mathrm{Ca}$} \\
\hline & $\Delta l c a \rightarrow \Delta l m l$ & 0.04397 & 0.8351 & No & \\
\hline & $\Delta l m l \rightarrow \Delta l c$ & 0.72594 & 0.3998 & No & \multirow[t]{2}{*}{$\mathrm{ml} \neq \mathrm{C}$} \\
\hline & $\Delta l c \rightarrow \Delta l m l$ & 0.05703 & 0.8126 & No & \\
\hline & $\Delta l c \rightarrow \Delta l y$ & 2.71658 & 0.1055 & Yes & \multirow[t]{2}{*}{$\mathbf{c} \leftrightarrow \mathrm{y}$} \\
\hline & $\Delta l y \rightarrow \Delta l c$ & 3.37360 & 0.0721 & Yes & \\
\hline & $\Delta l c a \rightarrow \Delta l c$ & 0.72594 & 0.3998 & No & \multirow[t]{2}{*}{$\mathrm{ca} \neq \mathrm{C}$} \\
\hline & $\Delta l c \rightarrow \Delta l c a$ & 0.05703 & 0.8126 & No & \\
\hline \multirow{12}{*}{ PAKISTAN } & $\Delta l m l \rightarrow \Delta l y$ & 0.07337 & 0.7876 & No & \multirow[t]{2}{*}{$\mathrm{ml} \neq \mathrm{y}$} \\
\hline & $\Delta l y \rightarrow \Delta \operatorname{lml}$ & 0.00509 & 0.9434 & No & \\
\hline & $\Delta l c a \rightarrow \Delta l y$ & 1.95281 & 0.1698 & No & \multirow[t]{2}{*}{$\mathrm{ca} \neq \mathrm{y}$} \\
\hline & $\Delta l y \rightarrow \Delta l c a$ & 0.18292 & 0.6711 & No & \\
\hline & $\Delta l m l \rightarrow \Delta l c a$ & 0.06188 & 0.8048 & No & \multirow[t]{2}{*}{$\mathrm{ca} \rightarrow \mathrm{m}$} \\
\hline & $\Delta l c a \rightarrow \Delta l m l$ & 2.71303 & 0.1055 & Yes & \\
\hline & $\Delta l m l \rightarrow \Delta l c$ & 0.08066 & 0.7776 & No & \multirow[t]{2}{*}{$\mathrm{ml} \neq \mathrm{C}$} \\
\hline & $\Delta l c \rightarrow \Delta \operatorname{lml}$ & 0.03048 & 0.8621 & No & \\
\hline & $\Delta l c \rightarrow \Delta l y$ & 2.18291 & 0.1457 & Yes & \multirow[t]{2}{*}{$\mathrm{c} \leftrightarrow \mathrm{y}$} \\
\hline & $\Delta l y \rightarrow \Delta l c$ & 5.48893 & 0.0231 & Yes & \\
\hline & $\Delta l c \rightarrow \Delta l c a$ & 0.80919 & 0.3736 & No & \multirow[t]{2}{*}{$\mathrm{ca} \neq \mathrm{C}$} \\
\hline & $\Delta l c a \rightarrow \Delta l c$ & 0.58718 & 0.4479 & No & \\
\hline
\end{tabular}

For India, it was determined the evidence of no causality for all variables with the difference of the MSBGC results. In the Brazil, it was found the evidence of unidirectional causality from Current account to real GDP, from current account to energy imports and from real GDP to energy imports. The results of one-way causality from current account to energy imports is consistent with the results of MSBGC test in the only regime 2 . On the other side, it was determined the evidence of no causality between militarization and real GDP, between militarization and current account, and between militarization and energy imports.

For Turkey, there is the evidence of none causality between militarization and real GDP, between militarization and current account, between militarization and energy imports, between current account and energy imports, and between current account and energy imports. As similar to the results of MSBGC 
test in regime 1 , it was determined the evidence of bidirecional causal relation between energy imports and economic growth. It is determined the evidence of the two-way causal nexus between 'dc' and 'dy' for Pakistan which is consistent with the consequences of MSBGC tests in regime 2 and 3 . As completely differentiation from the results of MSBGC test, it was found the evidence of no causality between militarization and real GDP, between current account and real GDP, between militarization and energy imports, between current account and energy imports.

\section{Conclusion and Policy Implications}

This paper aims at analyzing the relationship among current account balance, military imports, economic growth, and energy imports, from 1972 to 2017 in the diverse regimes of the economies in the India, Brazil, Turkey and Pakistan. For this aim, MSBVAR and MSBGC methods were used which allow to determine this relation in the diverse stages of the economy as growth and crisis stages for the selected countries. It is the first paper analyzed the relationship among current account balance, militarization, economic growth, and energy imports for the countries by using MSBVAR and MSBGC methods to appraise this relation.

According to MSBGC results, there is inter-relation between the variables. Policies that reduce energy imports and military expenditure improves the current account balance, however, these policies have adverse effects on GDP of the countries because of a causal relation between GDP and militarization. Militarization, energy imports, economic growth and current account balance relate to each other's. Energy imports increase in the effect of militarization races and economic growth since militarization leads to consumption of huge amounts of petroleum in planes, ships, and tanks. Since roughly three-quarters of the oil through the world are consumed by the military forces. In addition, the energy imports of the military was increased by the industries producing the equipment for military forces.

In a general manner, the energy imports and military expenditure as fiscal policy have effect on current account balance and economic growth. While the countries exporting the products of defense industry have benefited from multiplier effects, the importing countries are charged with the costs. The prices of energy and weapons have economic impacts for both importer and exporter countries. For energy-importers, an exogenous rise in the price of imported energy is frequently viewed as a negative trade shock over their special impacts on production decisions. The prices of imported weapons negatively affect the current account of balance.

The current account balance of the selected countries are vulnerable to the negative energy shocks and militarization. The economic growth will be forced to decrease since it is based deeply on imported raw-materials and intermediary goods, especially in the selected countries. The inflationary effects will become much more apparent during the shocks. The increased import prices bring on the load on the balance of the current account. As the countries reduce their dependency on energy and weapons imports, their trade or current account balances can improve while their sensitivity to sudden oil price shocks can decrease. As countries decrease their 
The Relation Between Growth, Energy Imports, Militarization and Current Account Balance in India, Brazil, Turkey and Pakistan

dependence on energy and weapons imports, this may reduce their current account imbalances, they can decrease their sensitivity to sudden energy price changes.

\section{REFERENCES}

[1] Hamilton, J.D. (2003), What Is an Oil Shock? Journal of Econometrics, 113(2): 363-398;

[2] Kilian, L., Rebucci, A., and Spatafora, N. (2009), Oil Shocks and External Balances. Journal of International Economics, 77(2): 181-194;

[3] Bildirici, M., Sonustun, F. O. (2018), The Effects of Oil and Gold Prices on OilExporting Countries. Energy strategy reviews 22: 290-302;

[4] Hamilton, J.D. (1989), A New Approach to the Economic Analysis of Nonstationary Time Series and the Business Cycle. Economerica, 57: 357-384; [5] Huntington, H.G. (2015), Crude Oil Trade and Current Account Deficits. Energy Economics, 50:70-79;

[6] Yi, K.M. (1993), Can Government Purchases Explain the Recent U.S. Net Export Deficits? Journal of International Economics, 35: 201-225;

[7] Custers, P. (2010), Military Keynesianism Today: An Innovative Discourse. Race and Class, 51(4): 79-94;

[8] Dunne, J. P., Nikolaidou, E. (2012), Defence Spending and Economic Growth in the EU15. Defence and Peace Economics, 23(6):537-548;

[9] Wu, Y.H., Ho, C.C. and Lin, E. (2017), Measuring the Impact of Military Spending: How Far Does a DSGE Model Deviate from Reality? Defence and Peace Economics, 28(5): 585-608;

[10] Bildirici, M. E., Bakirtaş, T. and Kayıkçı, F. (2012), Economic Growth and Electricity Consumption: Auto Regressive Distributed Lag Analysis. Journal of Energy in Southern Africa, 23: 29-45;

[11] Benoit, E. (1973), Defense and Economic Growth in Developing Countries. Boston: Lexington Books;

[12] Benoit, E. (1978), Growth and Defense in Developing Countries. Economic Development and Cultural Change, 262: 271-280;

[13] Jorgenson, A. K., Clark, B. and Givens, J.E. (2012), The Environmental Impacts of Militarization in Comparative Perspective: An Overlooked Relationship. Nature and Culture, 7(3): 314-337;

[14] Bildirici, M. (2016), Defense, Economic Growth and Energy Consumption in China. Procedia Economics and Finance, 38: 257-263; 
Melike Bildirici, Fazıl Kayıkçı

[15] Bildirici, M. (2017), CO2 emissions and militarization in G7 countries: panel cointegration and trivariate causality approaches, Environment and Development Economics, 22(6):771-791;

[16] Bildirici, M. (2017), Militarization, Economic Growth and Petroleum Consumption in Brazil, Russian, India, China, Turkey, South Africa and Mexico, Economic Computation and Economic Cybernetics Studies and Research, 51(2): 249266;

[17] Bildirici, M.E., Alp, E.A. and Bakirtas, T. (2010), The Great Recession and the Effects Of Oil Price Shocks And The U.S. Recession: A Markov-Switching And

TAR-VEC Analysis. The Journal of Energy Development, 352: 215-277;

[18] Hamilton, J.D. (2003), What Is an Oil Shock? Journal of Econometrics, 113(2):

363-398;

[19] Renner, M. (1991), Oil and the Golf War. Middle East Report, No. 17;

[20] SIPRI (2012), Year Book. Armaments, disarmament and international securtiy;

[21] Sims, C. A. and T. Zha (1998), Bayesian Methods for Dynamic Multivariate Models. International Economic Review, 39(4): 949-968;

[22] Akgul I., Bildirici, M.E. and Ozdemir, S. (2014), Evaluating the nonlinear linkage between gold prices and stock market index using Markov-Switching Bayesian VAR models, 4th International Conference on Leadership, Technology, Innovation and Business Management (ICLTIBM), İstanbul, Turkey, 19 - 21 Nov. 2014, 210 (408-415); [23] OECD (2017), Composite Leading Indicators: Turning Points of Reference Series and Component Series;

[24] ECRI (2016), International Business Cycle Dates, Business Cycle Chronologies. https://www.businesscycle.com/ecri-business-cycles/international-business-cycledates-chronologies, (accessed date: 09. 03. 2017). 